\title{
Maresin 1 Attenuates Ventilator-induced Lung Injury by Reducing Oxidative Stress through the Nrf2/HO-1 and NF-KB Pathways
}

\section{Fuquan Wang}

Huazhong University of Science and Technology

\section{Guangtao Pan}

Yancheng TCM Hospital, Nanjing University of Chinese Medicine

Jingxu Wang

Huazhong University of Science and Technology

Haifa Xia ( $\square$ haifaxia@163.com)

Huazhong University of Science and Technology

Shanglong Yao

Huazhong University of Science and Technology

Dingyu Zhang

Huazhong University of Science and Technology

\section{Ming Chen}

Huazhong University of Science and Technology

\section{Research Article}

Keywords: Maresin1, VILI, Nrf2, HO-1, NF-KB

Posted Date: August 13th, 2021

DOl: https://doi.org/10.21203/rs.3.rs-792978/v1

License: (9) This work is licensed under a Creative Commons Attribution 4.0 International License. Read Full License 


\section{Abstract}

Mechanical ventilation could support the lives of patients with respiratory failure in a variety of ways, including maintaining airway patency and improving oxygenation, etc. However, mechanical ventilation itself could lead to lung damage, which is called mechanical ventilation-related lung injury (VILI). The incidence of VILI is high and the prognosis is poor, so clarifying the mechanism of VILI and seeking effective preventive and therapeutic measures are the urgent medical problem to be resolved. By constructing the VILI model, we studied the effect of Maresin1 on VILI and explored its possible mechanism at the animal level. We tested the related indicators of lung injury, the oxidative stress response, and the inflammatory response. The results indicated that Maresin 1 could inhibit the oxidative stress response and excessive inflammation, thus ameliorating lung injury in VILI. We also detected the expression levels of the principal oxidative stress pathway Nrf2 / HO-1 and the key inflammatory transcription factor NF-KB. We used the HO-1 inhibitor ZnPP to further confirm our conclusion. Our results suggested for the first time that Maresin 1 could promote the activation of the Nrf2 / HO-1 pathway and significantly suppress the expression of NF-KB to exert a positive role in VILI.

\section{Introduction}

Mechanical ventilation is a vital means of life support for the patients who cannot rely on their spontaneous breathing function to meet the need of their normal life activities[1], such as the patients who are under general anesthesia and suffering from respiratory failure. However, while mechanical ventilation provides respiratory support and improves the oxygenation function of the body, mechanical ventilation itself could also cause or aggravate the damage of lung tissue, known as ventilator-induced lung injury (VILI). VILI could even evolve into acute respiratory distress syndrome (ARDS) and endanger the patient's life[2]. Though the protective lung ventilation strategies have made definite progress, VILI still poses challenges for the clinical practice[3]. Therefore, the search for new and effective treatments for VILI is an important problem to be solved urgently[4].

The excessive stretch stimulation of large tidal volume mechanical ventilation on the alveolar wall may cause obvious pathological damage including the alveolar-capillary exudation, the neutrophil infiltration, the alveolar hemorrhage, and the alveolar wall thickening, etc.[5] Many studies suggested that in addition to the mechanical damage to VILI, the oxidative stress and the excessive inflammatory response could also significantly increase the severity of VILI[6]. Therefore, controlling the excessive oxidative stress response and the inflammatory responses provide targets for the prevention and treatment of VILI.

Nuclear factor erythroid 2 related factor2 (Nrf2) could promote the transcription of heme oxygenase-1 (HO-1), the indirect metabolite of which has a strong antioxidant capacity, so Nrf-2 / HO-1 plays an important role in cell adaptation and antioxidant stress[7]. HO-1 is the rate-limiting enzyme in the heme metabolism pathway in the human body. As a protective protein, $\mathrm{HO}-1$ has numerous biological activities such as anti-oxidation, anti-inflammatory, anti-tracheal epithelial cell 
proliferation, anti-apoptosis, and regulation of microcirculation. $\mathrm{HO}-1$ could negatively regulate the inflammatory response and the oxidative stress response in acute lung injury[8].

NF-KB, as a target for many inflammatory diseases, is regulated by multiple signals. ROS, a vital production of oxidative stress, is considered to be one of the important regulators of NF-KB activation and plays an important role in the occurrence and development of inflammation[9]. ROS is necessary for the objects to play a role in eliminating bacteria, but overexpressed ROS could induce inflammation and results in tissue damage. Studies have shown that the activation of Nrf2 / HO-1 could inhibit the production of ROS induced by lipopolysaccharides (LPS) and reduce the expression of inflammatory mediators to play a protective role in improving lung injury[10,11].

Specialized pro-resolving mediators(SPM) such as maresins and resolvins, which are derived from polyunsaturated fatty acids, play an important role in controlling inflammation and oxidative stress[12, 13]. As a novel SPM[14], the effect of Maresin1 on improving lung injury through multiple mechanisms has been confirmed $[15,16]$. There was also a recent investigation shown that MaR1 could inhibit the oxidative stress in lung IRI[17]. However, whether MaR1 could inhibit the oxidative stress and the inflammation response to exert a protective role in VILI has not been reported.

Our previous studies have shown that Resolvin D1 could inhibit the expression of HMGB-1 via the Nrf2 / HO-1 pathway and thus play a positive role in VILI[18]. However, whether Maresin1 could reduce the oxidative stress response and the inflammatory response through the Nrf2 / HO-1 pathway and NF-KB have not been confirmed. Therefore, in this experiment, we chose the VILI model to verify whether Maresin 1 could inhibit the oxidative stress response and the inflammatory response in VILI and whether its related effects are exerted through the Nrf2 / HO-1 and the NF-KB pathways.

\section{Materials And Methods 2.1 Animals}

The 6-8 weeks old, weighing 20-22 g, SPF male C57BL/6 mice were purchased from the Experimental Animal Center of Wuhan University. The mice were randomly divided into 3 per cage and with free access to food and water. All the mice were housed in the animal experiment center of the SPF environment with the humidity of $60-65 \%$, the light-dark cycles $12 / 12 \mathrm{~h}$, and the temperature of $22-24^{\circ} \mathrm{C}$. All the mice were acclimatized for one week before the start of the experiment. The experiment was performed in accordance with the Chinese Animal Research Guidelines and was approved by the Laboratory Animal Management Committee of Tongji Medical College of Huazhong University of Science and Technology.

\subsection{Experimental design}


The construction method of the VILI model is the same as that described in our previous literature[18]. All experiments were repeated no less than three times.

The mice were first randomly divided into the following 4 groups $(n=7)$ : (1)The sham operation group (the Sham group): tracheal intubation was performed, but no mechanical ventilation was performed. $200 \mu$ of saline was injected into the tail vein 30 minutes before the tracheal intubation (2) The maresin1(Cayman Chemical, Ann Arbor, MI, USA) intervention control group》the MaR1 group》: tracheal intubation was performed, but no mechanical ventilation was performed. Tail vein injection of Maresin $11 \mathrm{ng}(200 \mu \mathrm{l}) 30$ minutes before the tracheal intubation; (3) The large tidal volume mechanical ventilation group (the HVT group): tidal volume $40 \mathrm{ml} / \mathrm{kg} ; 200 \mu \mathrm{l}$ of saline was injected into the tail vein 30 minutes before the tracheal intubation. (4) the Maresin1 intervention group \the MaR1 + HVT group): tidal volume $40 \mathrm{ml} / \mathrm{kg}$, tail vein injection of Maresin $11 \mathrm{ng}(200 \mu \mathrm{l}) 30$ minutes before the beginning of the tracheal intubation. After that, we used Znpp, the specific HO-1 inhibitor. The mice were randomly divided into the following six groups(n=7). (1) The Sham group(2) The MaR1 group (3) The ZnPP intervention control group $\nabla$ the

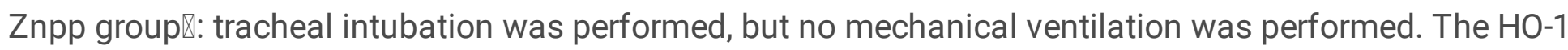
antagonist ZnPP (10 mg/kg) was intraperitoneally injected and $200 \mu \mathrm{l}$ of saline was injected into the tail vein 30 minutes before the tracheal intubation. (4) The HVT group (5) The MaR1 + HVT group (6) The HO1 antagonist zinc protoporphyrin + Maresin1 group (the ZnPP + Maresin1 group): ZnPP (10 mg/kg) was intraperitoneally injected 30 minutes before the start of the tracheal intubation and the rest of the treatment was consistent with the MaR1 + HVT group. The mice in the sham group, the MaR1 group, the HVT group, and the MaR1 + HVT group were intraperitoneally injected additional saline $(10 \mathrm{mg} / \mathrm{kg}) 30$ minutes before starting the experiment. The respiratory rate of each group of mechanical ventilation is 80 times/min, the mechanical ventilation time is $4 \mathrm{~h}, \mathrm{FiO} 2=2 \mathrm{l} \% \mathrm{I}: \mathrm{E}=1: 2, \mathrm{PEEP}=0 \mathrm{mmHg}$.

\subsection{Histological analysis of lung tissues}

The right upper lobe of the lung tissue was harvested for the pathological analysis. The lung tissue was made into slices about approximated $4 \mu \mathrm{m}$ thick after being fixed in $4 \%$ paraformaldehyde for $24 \mathrm{~h}$. The slices were stained with the hematoxylin and the eosin. Then the sections were observed under the ordinary optical microscope. The pathological injury score of the lung tissue was performed by using the lung tissue injury scoring criteria published by the American Thoracic Society[19].

\subsection{Lung wet-to-dry weight ratio}

The middle lobe of the right lung tissue from each group was collected to calculate the lung wet-to-dry weight ratio. After cleaning the surface of the fresh lung tissue with absorbent paper, weigh the initial weight of the lung tissue. Then put the lung tissue into the oven until the weight of the lung tissue becomes a constant weight. The weight of the lung tissue was weighed again. Calculate the lung wet-todry weight ratio and assess the extent of the lung tissue edema. 


\subsection{Analysis of arterial blood gas}

At the end of the experiment, the blood (heparin anticoagulant) of the left carotid artery was collected and the arterial partial pressure of oxygen was immediately measured by a blood gas analyzer (Radiometer, Copenhagen, Denmark).

\subsection{Measurement of the markers of the oxidative stress in lung tissues}

According to the manufacturer's instructions, the malondialdehyde (MDA), superoxide dismutase (SOD), catalase (CAT), glutathione (GSH), myeloperoxidase(MPO) and 15-F2t-isoprostane were detected by their corresponding commercial assay kits (Jiancheng Bioengineering Insti-

tute, Nanjing, China). The activities of these indicators were assessed by a spectrophotometer

\subsection{Bronchial alveolar lavage fluid analysis}

After the ligation of the right lung, $0.5 \mathrm{~mL}$ phosphate buffer saline (PBS) was utilized to lavage the left lung to obtain the BALF. The lavage process was repeated three times, and the recovery rate of more than $80 \%$ was considered effective.

The number of cells in the BALF was counted by a haematocytometer.

The protein concentration of the BALF was measured by using the BCA Protein Assay kit (Thermo Fisher Scientific).

According to the manufacturer's instructions, the corresponding murine ELISA kits (Dakewe Bioengineering Co., Ltd., Shenzhen, China) were used to evaluate the levels of the related inflammatory mediators (IL-6, TNF- $\alpha$, IL- $\beta$ ) in the BALF.

\subsection{Western blotting analysis}

According to the manufacturer's protocol, the protein of the lung tissue was extracted by using the protein extraction reagent kit (KeyGEN BioTECH, Nanjing, China) and the nucleoprotein was extracted using the Nuclear and Cytoplasmic Protein Extraction Kit(Beyotime, China). The protein concentration was measured with the BCA Protein Assay kit(Thermo Fisher Scientific). The western blotting analysis was performed as mentioned in our previous research[20]. The membrane was first incubated with the primary antibody: Nrf2 (Cell Signaling Technology, Danvers, MA, USA), HO-1(Cell Signaling Technology, Danvers, MA, USA), NF-kB p65(Cell Signaling Technology, Danvers, MA, USA), Histone 3 (Cell Signaling Technology, Danvers, MA, USA), GAPDH $₫$ Antgene, China $\ a t ~ 4^{\circ} \mathrm{C}$ overnight. Then the membrane was incubated with 
the corresponding secondary antibody (Cell Signaling Technology, Danvers, MA, USA). Finally, the enhanced chemiluminescence reagent (Beyotime Institute of Biotechnology, Shanghai, China) and the UVP 160 imaging system (Upland, CA, USA)was used for exposure imaging. The grayscale value of the images was analyzed using the Image $\mathrm{J}$ software.

\subsection{Statistical Analysis.}

The whole data were expressed as means $\pm S E M$. The processes of the statistical analysis process and the mapping were performed by the Graphpad Prism 6.0 software. One-way analysis of variance (ANOVA) was used for comparison among groups, and the differences between groups were compared using Newman-Keuls. $P<0.05$ was considered to be statistically significant.

\section{Results}

\subsection{Marsin1 attenuated ventilator-induced lung injury and significantly improves pulmonary function.}

The pathological changes of the lung tissue were assessed by HE staining (Figure 1A-D). The content of the lung injury in the sham group and the MaR1 group didn't show a significant difference, which indicated Maresin1 itself does not have obvious damage to the lung tissue. Excessive mechanical ventilation could cause evident lung injury and Maresin 1 could attenuate the histological lung injury. The pathological injury score(Figure 1E) was in line with the results observed by He staining.

The detection results of the arterial partial pressure of oxygen (Figure 1F), the W/D ratio(Figure 1G), and the protein concentration in BALF $($ Figure $1 \mathrm{H})$ were consistent with the results of the pathological changes. Maresin 1 itself had no significant effect on the partial arterial oxygen pressure, the W/D ratio, and the protein concentration in BALF. The PaO2 of the HVT group was significantly lower than the sham group $(P<0.01)$ while the W/D ratio and the protein concentration in BALF were significantly higher than the normal mice $(P<0.01)$, indicating that excessive mechanical ventilation could contribute to the lung injury. Compared with the HVT group, the PaO2 in the MaR1 + HVT group was significantly increased(P< $0.01)$, while the W/D ratio and the protein were significantly reduced $(P<0.01)$, suggesting that Maresin1 could reduce lung injury and improve lung oxygenation function.

\subsection{Maresin inhibited the excessive inflammatory response in VILI}

The excessive inflammation is the main cause that leads to the occurrence of the VILI. Our results $₫$ Figure

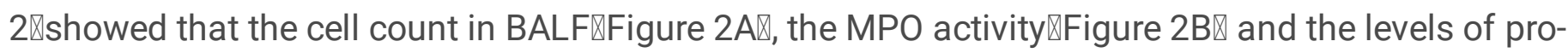
inflammatory factors $\triangle$ Figure $2 \mathrm{C}$-E囚 in the HVT group were significantly increased compared to the normal 
mice $(P<0.01)$, while Maresin1 significantly reduced the levels of pro-inflammatory factors, the cell count, and the MPO activity. These results suggested that excessive mechanical ventilation could cause a significant inflammatory response that leads to lung injury and Maresin 1 had a significant role in antiinflammatory.

\subsection{Maresin1 inhibited the oxidative stress response in VILI}

Oxidative stress plays an indispensable role in the initiation and progression of the VILI. As shown in Figure 3A-F, compared with the sham group, the HVT group showed low expression levels in GSH-PX and CAT but a higher level of the ROS, MDA, SOD, and15-F2t-isoprostane $(P<0.01)$. These tests revealed that excessive oxidative stress was indeed involved in VILI. Compared with the HVT group, the GSH-PX and CAT levels of the MaR1 + HVT group were higher $(P<0.01)$, and the ROS, MDA, SOD, and15-F2tisoprostane levels were lower $(P<0.01)$, clearing that Maresin could significantly inhibit the oxidative stress response.

\subsection{Maresin1 promote the activation of the Nrf2/HO-1 pathway and inhibited the NF-KB.}

The level of the protein expression level of the Nrf2, Histone 3, HO-1, GAPDH, and NF-KB p65 protein in the lung tissues were analyzed by Western blotting to clarify the possible mechanism by which Maresin1 ameliorates the VILI. The results (Figure4 A-D) suggested that Maresin1 could significantly increase the protein expression level of $\mathrm{HO}-1$ and the level of $\mathrm{Nrf} 2$ in the nucleus, at the same time, the expression of NF-KB p65 could be significantly inhibited by Maresin1. Furthermore, the inhibitor of HO-1, ZNPP, could significantly inhibit the effect of Maresin1(Figure4 E-H) on Nrf2/HO-1 and NF-KB p65. ZnPP reduced the expression of HO-1 and Nrf2 and increased the expression of NF-KB.

\subsection{ZnPP inhibited the protective effect of Maresin1 on lung tissue in VILI}

As shown in Figure 5, there were no obvious pathological changes that occurred in the sham group, the MaR1 group, and the ZnPP group, indicating that Maresin1 and ZnPP do not cause lung injury by themselves. Pathological sections of lung tissue in the HVT group showed noticeable damage to lung tissue compared with the sham group $(P<0.01)$. The pathological changes in the lung caused by HVT became not obvious after the treatment of Maresin1. However, the histological changes in the lungs of the ZnPP + Maresin1 group were obviously more severe than those in the MaR1 + HVT group $(P<0.01)$. The lung injury score accorded with the pathohistological change. These results indicated that Maresin1 decreased the pathological damage of lung tissue caused by mechanical ventilation in part by increasing the expression of $\mathrm{HO}-1$ 


\subsection{ZnPP weakened the inhibition effect of Maresin1 on the oxidative stress and inflammatory response.}

As shown in Figure 6, the MPO, the IL-1 3 , the TNF-a, the ROS, the MDA, and the 15-F2t-isoprostane in the HVT group were significantly higher than those in the sham group $(P<0.01)$. Maresin 1 could inhibit the expression of the factors referred to above $(\mathrm{P}<0.01)$. When the expression of HO-1 was inhibited by ZnPP, the MPO, the IL-1 $\beta$, the TNF-a, the ROS, the MDA, and the 15-F2t-isoprostane showed a significant increase compared with the MaR1 + HVT group $(P<0.01)$. These phenomena indicated that ZnPP could weaken the inhibitory effect of Maresin1 on the inflammation response and the oxidative stress response.

\section{Discussion}

As a common clinical treatment method, mechanical ventilation was widely used in clinical critical illness such as acute lung injury (ALI) and acute respiratory distress syndrome (ARDS). However, the current reports suggested that the prevalence of VILI in surgical patients with normal lung function is between $6.2 \%$ and $24 \%[21,22]$. For patients with pulmonary dysfunction, the incidence of VILI is higher. Various known pathophysiological factors including inappropriate inflammatory response, excessive destruction of airway barrier, alveolar edema, cell apoptosis, etc. could contribute to the occurrence of the VILI, but the precise pathological mechanism of VILI has not yet been elucidated.

Although the research on the prevention and treatment of VILI has made certain progress, such as small tidal volume ventilation, the application of related drugs and biological agents[23, 24], the incidence of VILI remains remarkable and exploring the exact and effective treatment for VILI are still medical problems to be solved urgently.

There were studies have shown that Maresin 1 could ameliorate the lung ischemia/reperfusion injury by suppressing the oxidative stress via activating the Nrf-2-mediated HO-1 signaling pathway[17, 25]. However, there is no research indicating whether Maresin 1 could mitigate lung injury by suppressing the oxidative stress in VILI. So we constructed the VILI model to clarify the role of the Maresin 1 exerts in VILI and explore and the possible mechanism and treatment methods of VILI. Our previous research showed that Maresin 1 could inhibit the excessive inflammation caused by sepsis in mice[20,26]in a dose-dependent manner, the $1 \mathrm{ng}$ dose of Maresin1 can significantly protect mice and is a very suitable dose choice. There was also related study shown that $1 \mathrm{ng}$ of Maresin 1 can effectively inhibit the oxidative stress response in lung tissue[17]. What's more, we chose two doses of Maresin 1 of $0.1 \mathrm{ng}$ and $1 \mathrm{ng}$ for pre-experiment. The pathological results of the lung showed that the protective effect of $1 \mathrm{ng}$ of Maresin1 on VILI was more stable and effective, so we chose $1 \mathrm{ng}$ of Maresin for research.

We first verified the protective effect of Maresin1 in VILI. The results of the pathological section showed that Maresin 1 could significantly ameliorate the extent of the pathological damage of lung tissue in VILI. Then we used the number of the cells in BALF to reflect the overall inflammation level, the protein concentration in BALF to assess the vascular permeability, and the changes in lung tissue W / D ratio to 
measure the severity of the pulmonary edema. The large exudation of capillary fluid and protein is the main cause of pulmonary edema[27]. Our results showed that Maresin 1 could mitigate the pathological damage of lung tissue, reduce the permeability of microvascular, and extenuate the degree of pulmonary edema in VILI. In addition, Maresin1 could dramatically improve lung oxygenation function.

Considering the important role of inflammatory response in VILI, we tested the relevant inflammatory indicators. The inflammatory mediators closely related to VILI are mainly MPO, TNF-a, IL-1 $\beta$, IL-6, etc[28]. MPO is a specific marker of PMN[19]. Our results showed that mice could develop severe inflammation after hyperventilation. The VILI mice showed a huge increase in related inflammatory indicators, such as cell counts in BALF and the levels of related inflammatory factors (eg IL-1 $\beta, I L-6$, TNF-a). Maresin 1 could markedly inhibit the inflammation response.

As we mentioned earlier, oxidative stress and inflammation are closely related, both played a vital role in the start and development of VILI. So we also explored the oxidative stress response in VILI. When oxidative stress occurs, the imbalance between oxidation and antioxidants of the body could be reflected by the measurement of the levels of a number of related indicators. Superoxide dismutase (SOD), catalase (CAT), and glutathione peroxidase (GSH-PX) could prevent lung injury from ROS exposure[29]. MDA could reflect the degree of lipid peroxidation in the body, $15 \mathrm{~F} 2 \mathrm{t}$ isoprostane is a highly specific and sensitive indicator of lipid peroxidation products in the body. Therefore, we tested the above indicators in lung tissue, and the results showed that mechanical ventilation could significantly worsen the oxidative stress response, and Maresin1 could inhibit the oxidative stress response.

We next studied the classical pathway of oxidative stress Nrf2 / HO-1 and the important inflammation response transcription factor NF-KB. Under normal physiological conditions, Nrf2 combined with keap1 and localized in the cytoplasm. When the oxidative stress response occurs, Nrf2 dissociates from the conjugate, translocates into the nucleus, and induces HO-1 transcription, thereby exerting antioxidant effects[30]. Uncontrolled oxidative stress response could also contribute to the activation of the NF$\mathrm{KB}[31]$. Under normal physiological conditions, the $\mathrm{p} 65$ subunit of NF-KB in the cytoplasm combines with IKBa to form the NF-KB - IKBa complex[32]. When cells are stimulated by oxidative stress, IKBa is phosphorylated and bound to ubiquitin to degrade. The degradation of $\mathrm{IKBa}$ results in that NF-KB is activated to bind to specific DNA sequences in the nucleus, thereby participating in the process of the inflammation responses[33]. So we examined the protein expression of $\mathrm{HO}-1$ and the expression of $\mathrm{Nrf} 2$ and NF-KB in the nucleus after the Maresin 1 treatment. The results showed that Maresin1 could promote the Nrf2 to translocate into the nucleus and significantly inhibit the translocation of NF-KB. To confirm our findings, we further used the HO-1 antagonist ZnPP. After the treatment of ZnPP, the expression levels of HO-1 and Nrf2 were drastically reduced, while the expression of NF-KB was significantly increased, indicating that Maresin1could indeed inhibit excessive oxidative stress to improve lung injury in VILI by activating Nrf2 / HO-1pathway.

To further verify that Maresin1 does exert antioxidant and anti-inflammatory effects through the Nrf2 / HO-1 pathway and NF-kB, we continued to use ZnPP to validate our hypothesis. As expected, when ZnPP 
was used to inhibit the expression of $\mathrm{HO}-1$, the degree of pathological damage in the lung tissue was significantly increased, and the blood oxygen partial pressure was considerably reduced. At the same time, we re-examined the relevant indicators of the oxidative stress response and the inflammation response. The results showed that when HO-1 was inhibited, the oxidative stress response and the inflammation response were significantly worsened. All these results indicated that Maresin 1 significantly inhibited the production of pro-inflammatory factors via the suppression of the NF-KB and exerted the antioxidative effects via promoting the activation of the HO-1/Nrf2 pathway.

\section{Conclusion}

Our study showed that Maresin1 could reduce the oxidative stress response and the inflammation response through the Nrf-2 / HO-1 and the NF-KB signaling pathway, thereby significantly improving VILI.

\section{Abbreviations}

Acute lung injury (ALI); Acute respiratory distress syndrome (ARDS) ; Catalase (CAT); Glutathione peroxidase (GSH-PX); The transcription of heme oxygenase-1 (HO-1); Nuclear factor erythroid 2 related factor2 (Nrf2) ;Superoxide dismutase (SOD);Specialized pro-resolving mediators(SPM) Lipopolysaccharides (LPS); Mechanical ventilation-related lung injury (VILI);

\section{Declarations}

\section{Ethics approval and consent to participate}

The experiment was performed in accordance with the Chinese Animal Research Guidelines and was approved by the Laboratory Animal Management Committee of Tongji Medical College of Huazhong University of Science and Technology.

\section{Consent for publication}

All authors knew and agreed to publish.

\section{Availability of data and material}

Data and materials can be obtained by contacting the corresponding author.

\section{Competing interests}

The authors declare no competing financial interests. 


\section{Funding}

This study was supported by the grants from the National Natural Science Foundation of China 『No.81701887凶.

\section{Authors' contributions:}

F.W. and G.P. wrote this article and performed the experiments. M.C. and J.W. established the animal models and collected samples. G.P. and F.W. analysed the data. J.W.and M.C. prepared the figures. H.X.,S.Y. and D.Z. designed the experiments and revised the article.

\section{Acknowledgments $\llbracket$}

Not applicable

\section{References}

1. Beitler JR, Malhotra A, Thompson BT: Ventilator-induced Lung Injury. Clin Chest Med 2016, 37(4):633-646.

2. Matthay MA, Ware LB, Zimmerman GA: The acute respiratory distress syndrome. The Journal of clinical investigation 2012, 122(8):2731-2740.

3. Gattinoni L, Marini JJ, Collino F, Maiolo G, Rapetti F, Tonetti T, Vasques F, Quintel M: The future of mechanical ventilation: lessons from the present and the past. Critical care (London, England) 2017, 21(1):183.

4. Salman D, Finney SJ, Griffiths MJ: Strategies to reduce ventilator-associated lung injury (VALI). Burns: journal of the International Society for Burn Injuries 2013, 39(2):200-211.

5. Slutsky AS, Ranieri VM: Ventilator-induced lung injury. 2013, 369(22):2126.

6. Tasaka S, Amaya F, Hashimoto S, Ishizaka A: Roles of oxidants and redox signaling in the pathogenesis of acute respiratory distress syndrome. Antioxid Redox Signal 2008, 10(4):739-753.

7. Loboda A, Damulewicz M, Pyza E, Jozkowicz A, Dulak J: Role of Nrf2/HO-1 system in development, oxidative stress response and diseases: an evolutionarily conserved mechanism. Cell Mol Life Sci 2016, 73(17):3221-3247.

8. Wei CC, Kong YY, Li GQ, Guan YF, Wang P, Miao CY: Nicotinamide mononucleotide attenuates brain injury after intracerebral hemorrhage by activating Nrf2/HO-1 signaling pathway. Sci Rep 2017, 7(1):717.

9. Qi S, Xin Y, Guo Y, Diao Y, Kou X, Luo L, Yin Z: Ampelopsin reduces endotoxic inflammation via repressing ROS-mediated activation of PI3K/Akt/NF-kappaB signaling pathways. International immunopharmacology 2012, 12(1):278-287. 
10. Wu D, Wang Y, Zhang H, Du M, Li T: Acacetin attenuates mice endotoxin-induced acute lung injury via augmentation of heme oxygenase-1 activity. Inflammopharmacology 2018, 26(2):635-643.

11. Tsai CL, Lin YC, Wang HM, Chou TC: Baicalein, an active component of Scutellaria baicalensis, protects against lipopolysaccharide-induced acute lung injury in rats. J Ethnopharmaco/2014, 153(1):197-206.

12. Francos-Quijorna I, Santos-Nogueira E, Gronert K, Sullivan AB, Kopp MA, Brommer B, David S, Schwab JM, López-Vales R: Maresin 1 Promotes Inflammatory Resolution, Neuroprotection, and Functional Neurological Recovery After Spinal Cord Injury. The Journal of neuroscience: the official journal of the Society for Neuroscience 2017, 37(48):11731-11743.

13. Gao Y, Zhang H, Luo L, Lin J, Li D, Zheng S, Huang H, Yan S, Yang J, Hao Y et al: Resolvin D1 Improves the Resolution of Inflammation via Activating NF- $\mathrm{B}$ p50/p50-Mediated Cyclooxygenase-2 Expression in Acute Respiratory Distress Syndrome. Journal of immunology (Baltimore, Md: 1950) 2017, 199(6):2043-2054.

14. Serhan CN, Yang R, Martinod K, Kasuga K, Pillai PS, Porter TF, Oh SF, Spite M: Maresins: novel macrophage mediators with potent antiinflammatory and proresolving actions. The Journal of experimental medicine 2009, 206(1):15-23.

15. Jie G, Hong L, Jing W, Hong Q, You S: Maresin 1 Prevents Lipopolysaccharide- Induced Neutrophil Survival and Accelerates Resolution of Acute Lung Injury. Shock 2015, 44(4):371.

16. Fullerton JN, Gilroy DW: Resolution of inflammation: a new therapeutic frontier. Nature reviews Drug discovery 2016, 15(8):551-567.

17. Sun Q, Wu Y, Zhao F, Wang J: Maresin 1 Ameliorates Lung Ischemia/Reperfusion Injury by Suppressing Oxidative Stress via Activation of the Nrf-2-Mediated HO-1 Signaling Pathway. Oxidative medicine and cellular longevity 2017, 2017:9634803.

18. Sun Z, Wang F, Yang Y, Wang J, Sun S, Xia H, Yao S: Resolvin D1 attenuates ventilator-induced lung injury by reducing HMGB1 release in a HO-1-dependent pathway. International immunopharmacology 2019, 75:105825.

19. Matute-Bello G, Downey G, Moore BB, Groshong SD, Matthay MA, Slutsky AS, Kuebler WM: An official American Thoracic Society workshop report: features and measurements of experimental acute lung injury in animals. American journal of respiratory cell and molecular biology 2011, 44(5):725-738.

20. Sun S, Wang J, Wang J, Wang F, Yao S, Xia H: Maresin 1 Mitigates Sepsis-Associated Acute Kidney Injury in Mice via Inhibition of the NF-kappaB/STAT3/MAPK Pathways. Front Pharmacol 2019, 10:1323.

21. Villar J, Blanco J, Kacmarek RM: Current incidence and outcome of the acute respiratory distress syndrome. Current Opinion in Critical Care 2015, 22(1):1.

22. Vm ADTF, Ranieri: Acute Respiratory Distress Syndrome: The Berlin Definition. Jama, 307.

23. Serpa Neto A, Cardoso SO, Manetta JA, Pereira VG, Esposito DC, Pasqualucci Mde O, Damasceno $\mathrm{MC}$, Schultz MJ: Association between use of lung-protective ventilation with lower tidal volumes and 
clinical outcomes among patients without acute respiratory distress syndrome: a meta-analysis. Jama 2012, 308(16):1651-1659.

24. Futier E, Constantin JM, Paugam-Burtz C, Pascal J, Eurin M, Neuschwander A, Marret E, Beaussier M, Gutton C, Lefrant JY et al: A trial of intraoperative low-tidal-volume ventilation in abdominal surgery. The New England journal of medicine 2013, 369(5):428-437.

25. Lv C, Jin Q: Maresin-1 Inhibits Oxidative Stress and Inflammation and Promotes Apoptosis in a Mouse Model of Caerulein-Induced Acute Pancreatitis. Medical science monitor: international medical journal of experimental and clinical research 2019, 25:8181-8189.

26. Gong J, Wu ZY, Qi H, Chen L, Li HB, Li B, Yao CY, Wang YX, Wu J, Yuan SY et al: Maresin 1 mitigates LPS-induced acute lung injury in mice. British journal of pharmacology 2014, 171(14):3539-3550.

27. Jerng JS, Hsu YC, Wu HD, Pan HZ, Wang HC, Shun CT, Yu CJ, Yang PC: Role of the renin-angiotensin system in ventilator-induced lung injury: an in vivo study in a rat model. Thorax 2007, 62(6):527535.

28. Driessche WV, Kreindler JL, Malik AB, Margulies S, Kim KJ: Interrelations/cross talk between transcellular transport function and paracellular tight junctional properties in lung epithelial and endothelial barriers. Ajp Lung Cellular \& Molecular Physiology 2007, 293(3):L520-524.

29. Kuo MY, Liao MF, Chen FL, Li YC, Yang ML, Lin RH, Kuan YH: Luteolin attenuates the pulmonary inflammatory response involves abilities of antioxidation and inhibition of MAPK and NFkappaB pathways in mice with endotoxin-induced acute lung injury. Food and chemical toxicology: an international journal published for the British Industrial Biological Research Association 2011, 49(10):2660-2666.

30. Bellezza I, Giambanco I, Minelli A, Donato R: Nrf2-Keap1 signaling in oxidative and reductive stress. Biochimica et biophysica acta Molecular cell research 2018, 1865(5):721-733.

31. Seo JY, Kim H, Seo JT, Kim KH: Oxidative stress induced cytokine production in isolated rat pancreatic acinar cells: effects of small-molecule antioxidants. Pharmacology 2002, 64(2):63-70.

32. Beg AA, Ruben SM, Scheinman RI, Haskill S, Rosen CA, Baldwin AS, Jr.: I kappa B interacts with the nuclear localization sequences of the subunits of NF-kappa B: a mechanism for cytoplasmic retention. Genes Dev 1992, 6(10):1899-1913.

33. Akira S, Takeda K: Toll-like receptor signalling. Nat Rev Immunol 2004, 4(7):499-511.

\section{Figures}



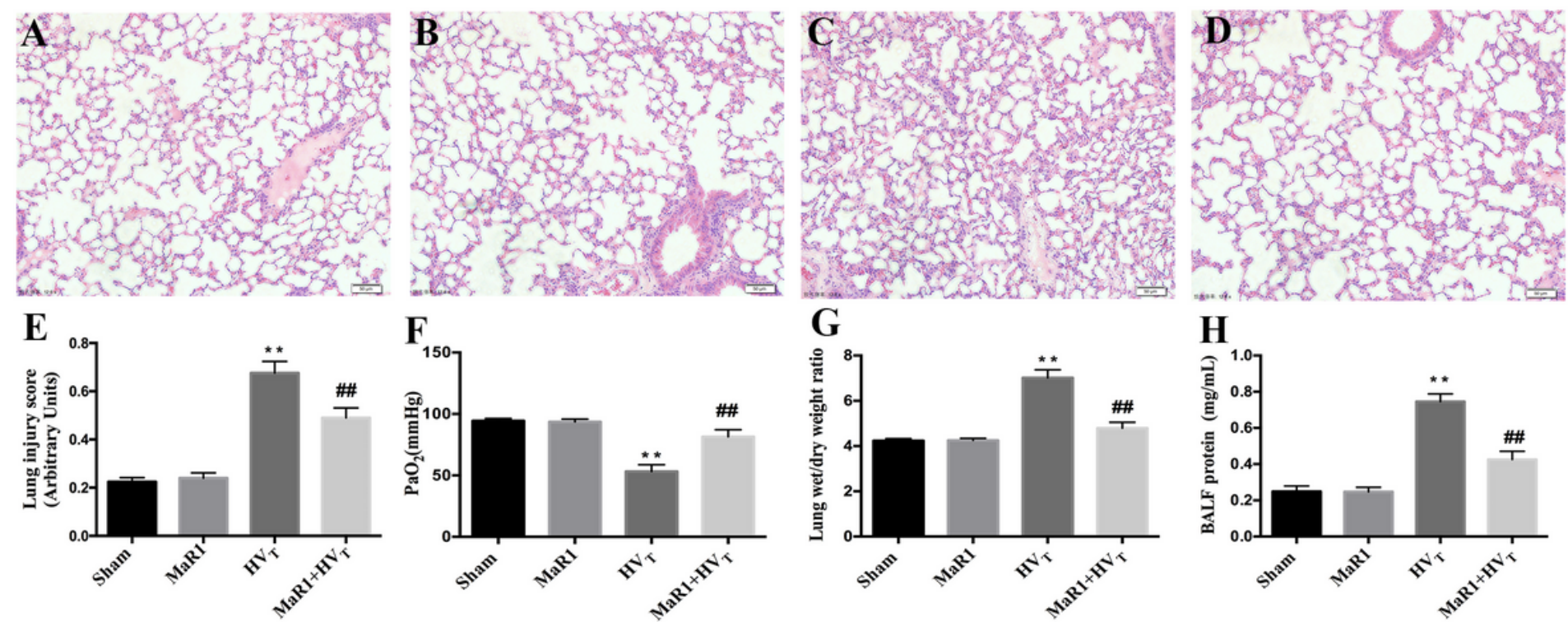

Figure 1

Maresin1 significantly improved the degree of lung injury in the VILI model. Fig2A-D are the representative pictures of $\mathrm{H}-\mathrm{E}$ staining lung tissues (magnification 200x). A: The sham group B: The MaR1 group C: The HVT group D: The MaR1 + HVT group. E: The lung injury scores of all groups. F: The arterial partial pressure of oxygen $\mathrm{G}$. The lung wet/dry weight ratio. $\mathrm{H}$. The protein concentration in BALF. Data was presented as means $\pm S E M, n=7 .{ }^{*} P<0.01$ versus the sham group; $\# \# P<0.01$ versus the HVT group.
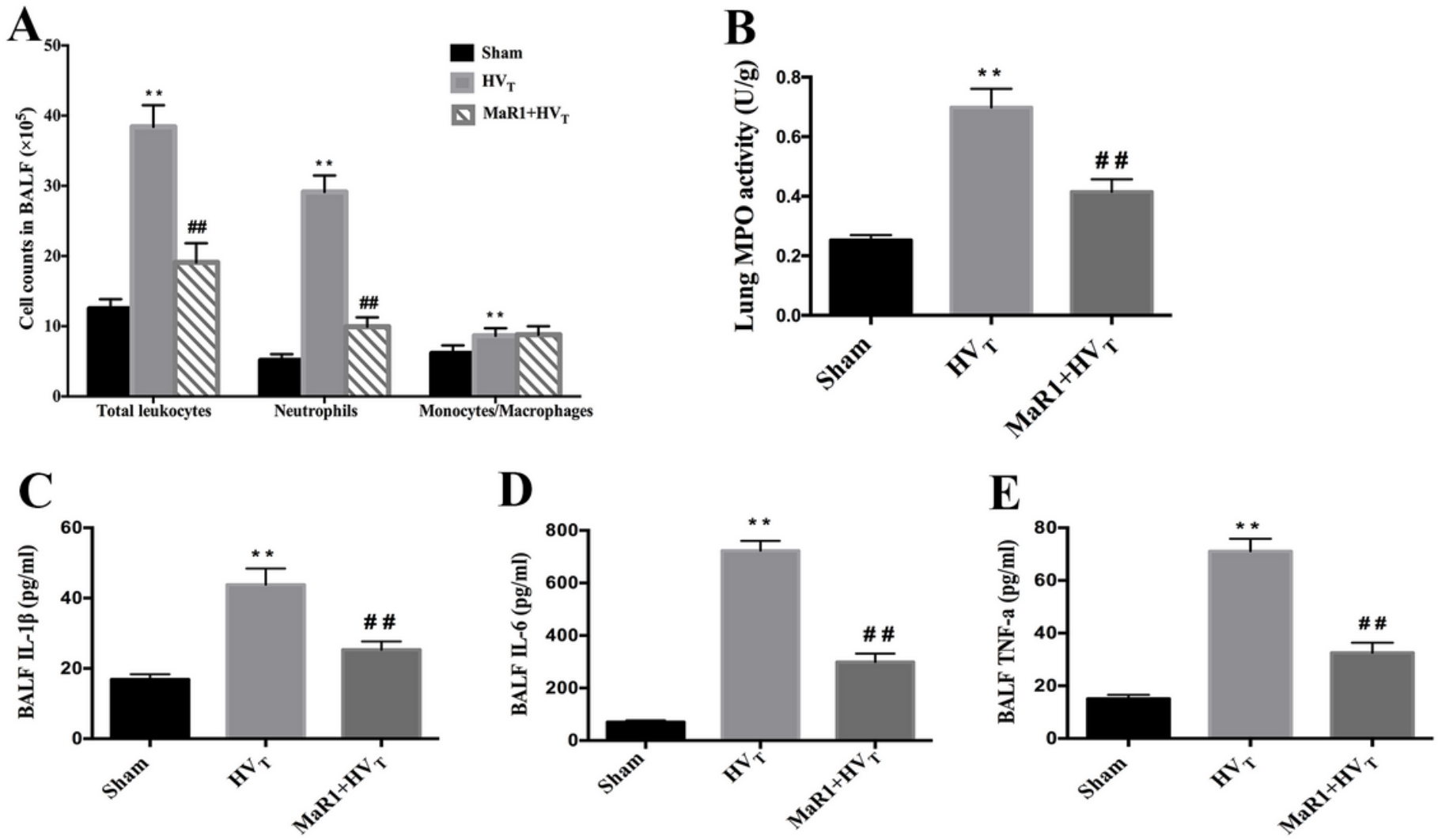

Figure 2 
Maresin1 inhibited the excessive inflammation response in VILI. A: The cell count in BALF B: The MPO activity C: IL-1 $\beta$ D: IL-6 E: TNF-a. Data was presented as means $\pm S E M, n=7$. ${ }^{*} P<0.01$ versus the sham group; \#\#P $<0.01$ versus the HVT group.
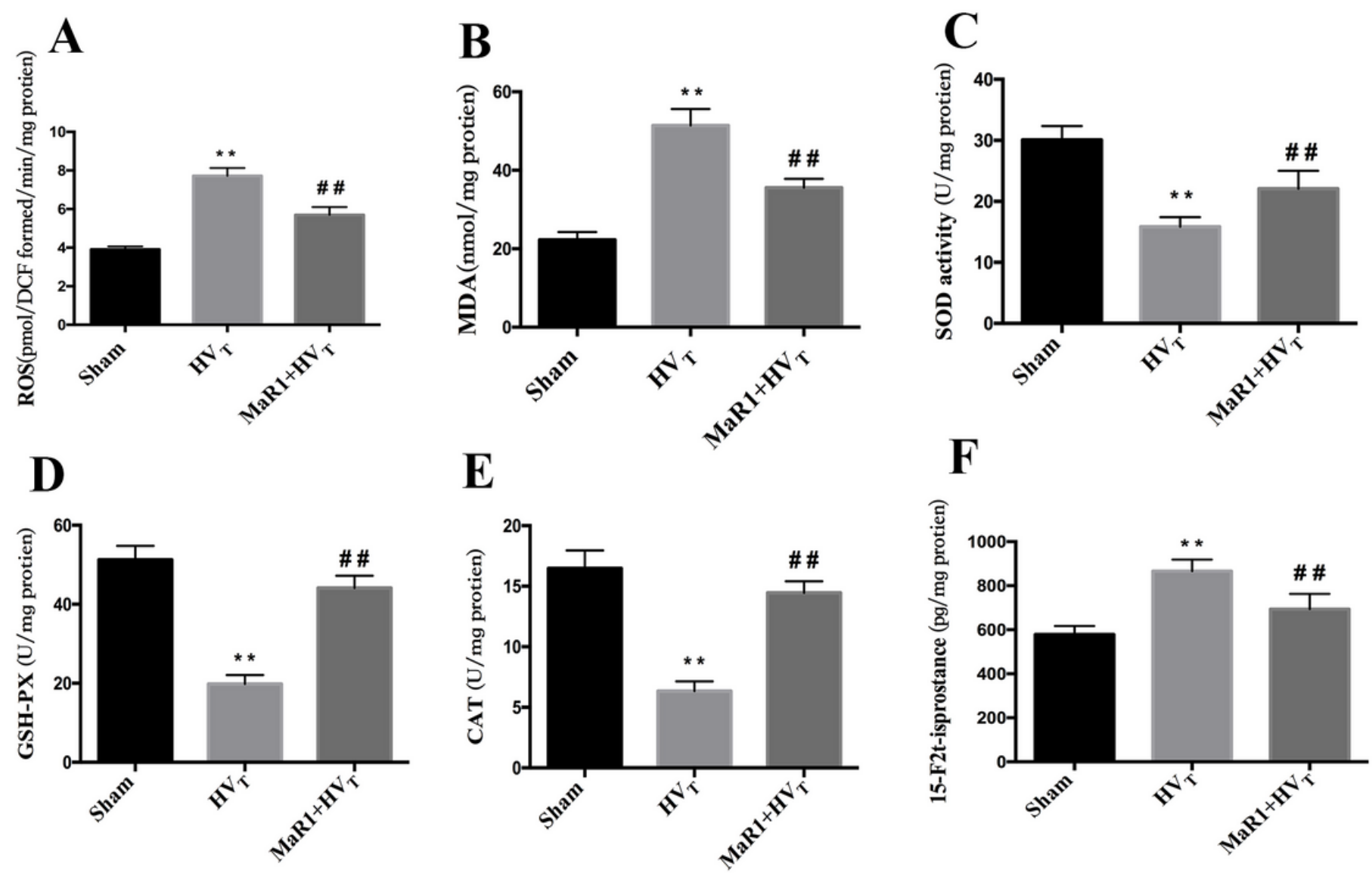

Figure 3

Maresin1 significantly inhibited the oxidative stress response in VILI. A: ROS B: MDA C: SOD D: GSH-PX E: CAT F: 15 -F2t-isoprostane Data was presented as means $\pm S E M, n=7 .{ }^{*} P<0.01$ versus the sham group; $\# \# P<0.01$ versus the HVT group. 
A

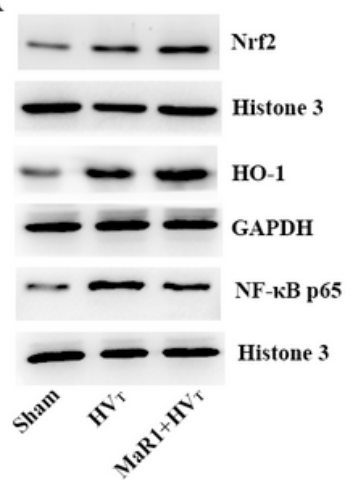

$\mathbf{E}$

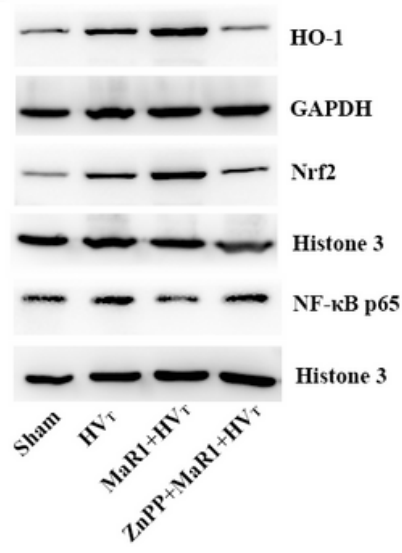

B

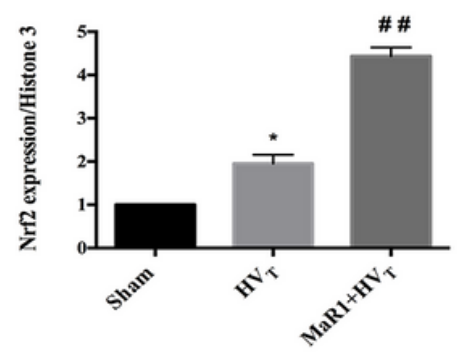

$\mathbf{F}$

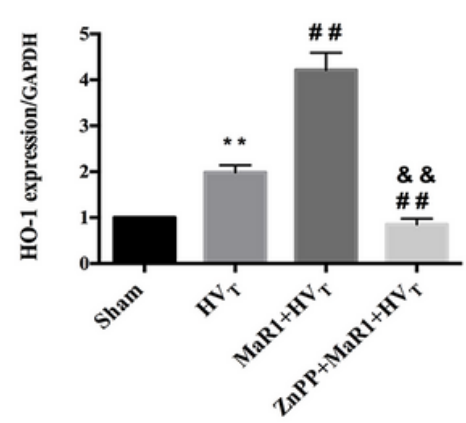

C

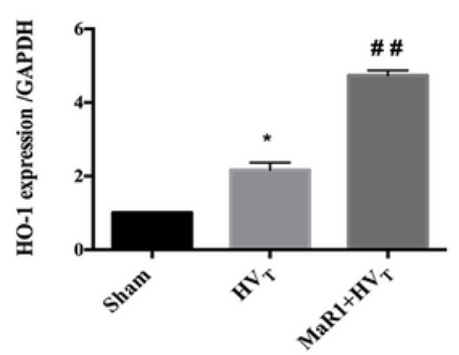

G

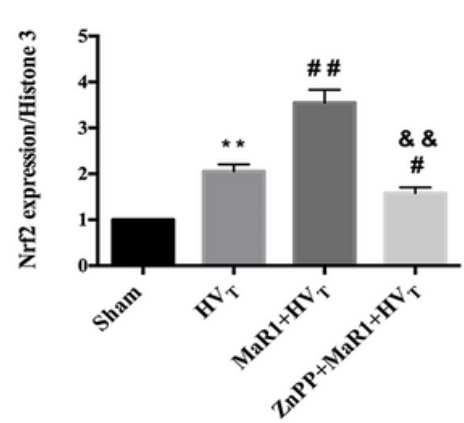

D

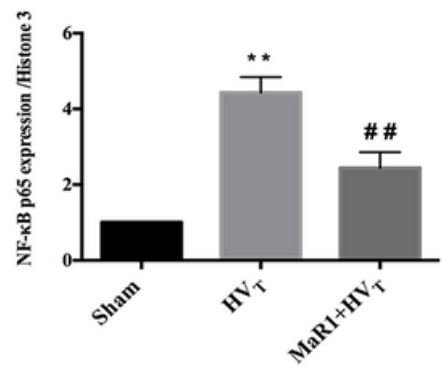

$\mathbf{H}$

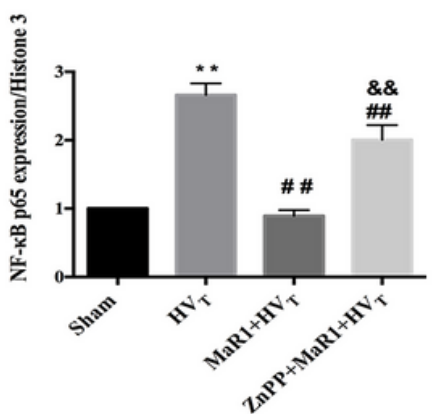

Figure 4

Maresin1 activated the Nrf2/HO-1 pathway and suppressed the NF-KB in VILI. A and E are the expression level of Nrf2, Histone 3, HO-1, GAPDH, and NF-KB p65 proteins of the lung tissues. B-D and F-H are the statistical tables corresponding to $A$ and $E$ respectively. Data was presented as means $\pm S E M, n=7$. *P< 0.05 versus the sham group; ${ }^{\star} \mathrm{P}<0.01$ versus the sham group; $\# \mathrm{P}<0.05$ versus the CLP group; $\# \# \mathrm{P}<$ 0.01 versus the CLP group; $\& \& P<0.01$ versus the MaR1 + HVT group. 

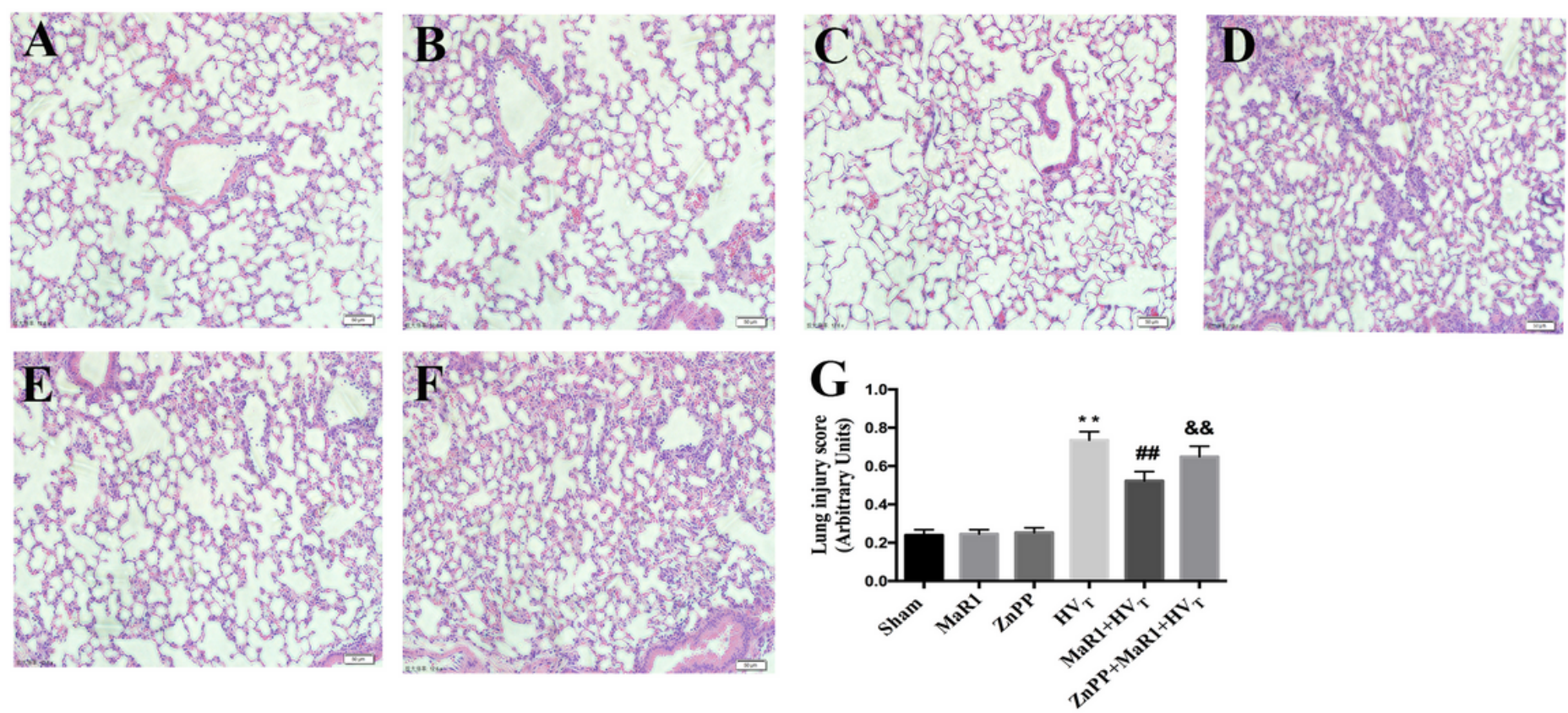

\section{Figure 5}

ZnPP dampened the protective effect of Maresin1 on lung tissue in VILI. Fig6A-F are the representative pictures of $\mathrm{H}-\mathrm{E}$ staining lung tissues (magnification 200x). A: The sham group B: The MaR1 group C: The ZnPP group D: The HVT group E: The MaR1 + HVT group. F: The ZnPP + Maresin1 group G: Lung injury scores of all the groups. Data was presented as means $\pm S E M, n=7$. $* * P<0.01$ versus the sham group; $\# \# P<0.01$ versus the HVT group $; \& \& P<0.01$ versus the MaR1 + HVT group. 

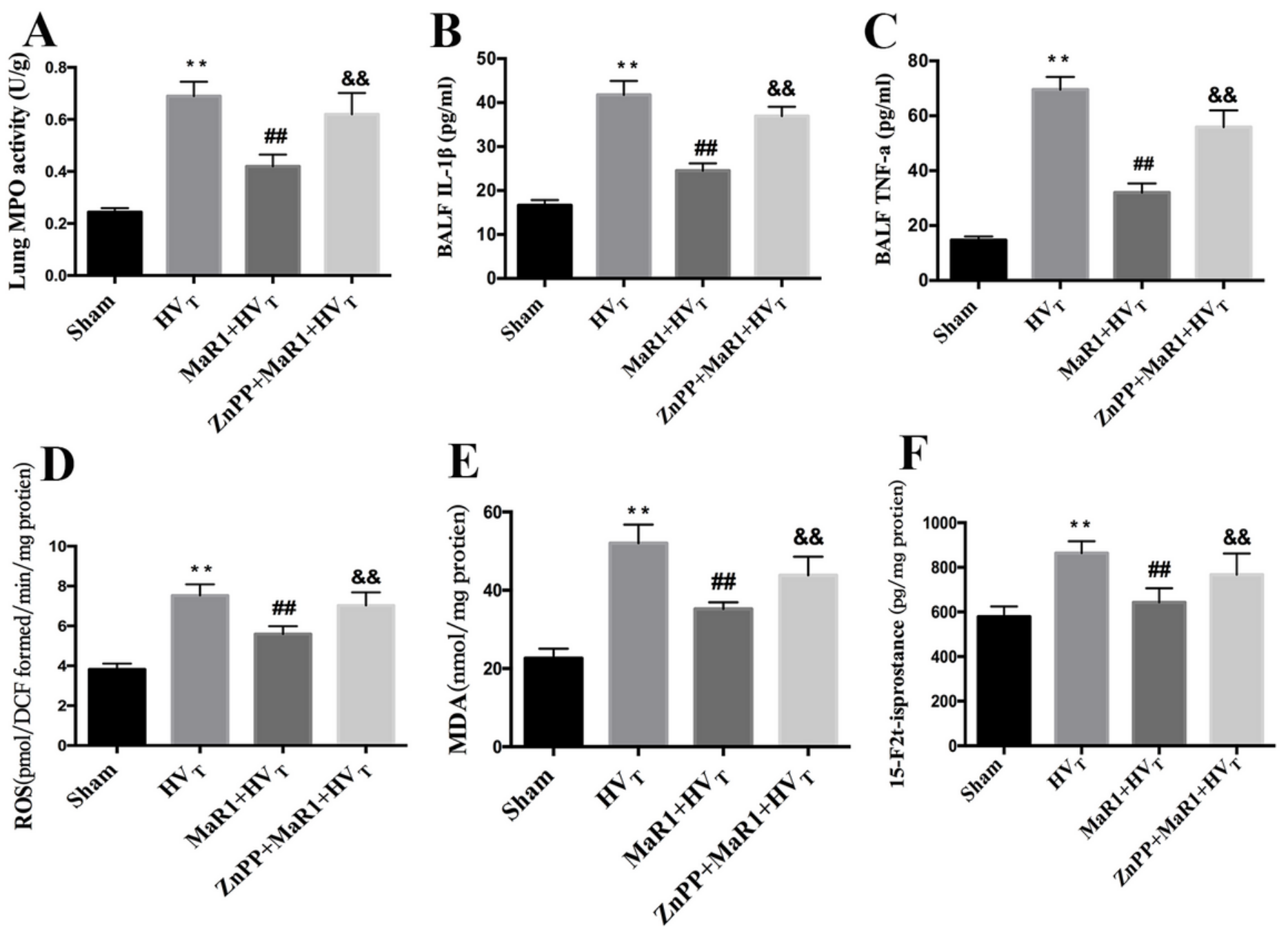

Figure 6

ZnPP weakened the inhibition effect of Maresin 1 on the inflammation response and the oxidative stress response. A: MPO activity B: IL-1 $\beta$ C: TNF-a D: ROS E: MDA F: 15-F2t-isoprostane. Data was presented as means $\pm S E M, n=7 .{ }^{* \star P}<0.01$ versus the sham group; \#\#P<0.01 versus the HVT group;\&\& $P<0.01$ versus the MaR1 + HVT group. 